\title{
The Connection between Gamma-Ray Bursts and Extremely Metal-Poor Stars as Nucleosynthetic Probes of the Early Universe
}

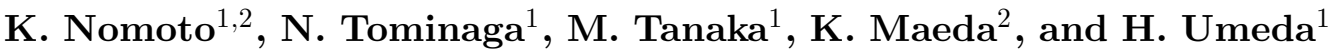 \\ ${ }^{1}$ Department of Astronomy, University of Tokyo, Bunkyo-ku, Tokyo 113-0033, Japan \\ email: nomoto@astron.s.u-tokyo.ac.jp \\ ${ }^{2}$ Institute for the Physics and Mathematics of the Universe, University of Tokyo, Kashiwa, \\ Chiba 277-8582, Japan
}

\begin{abstract}
The connection between the long GRBs and Type Ic Supernovae (SNe) has revealed the interesting diversity: (i) GRB-SNe, (ii) Non-GRB Hypernovae (HNe), (iii) X-Ray Flash (XRF)-SNe, and (iv) Non-SN GRBs (or dark HNe). We show that nucleosynthetic properties found in the above diversity are connected to the variation of the abundance patterns of extremely-metal-poor (EMP) stars, such as the excess of $\mathrm{C}$, Co, Zn relative to Fe. We explain such a connection in a unified manner as nucleosynthesis of hyper-aspherical (jet-induced) explosions of Pop III core-collapse SNe. We show that (1) the explosions with large energy deposition rate, $\dot{E}_{\mathrm{dep}}$, are observed as GRB-HNe and their yields can explain the abundances of normal EMP stars, and (2) the explosions with small $\dot{E}_{\text {dep }}$ are observed as GRBs without bright SNe and can be responsible for the formation of the C-rich EMP (CEMP) and the hyper metal-poor (HMP) stars. We thus propose that GRB-HNe and the Non-SN GRBs (dark HNe) belong to a continuous series of $\mathrm{BH}$-forming massive stellar deaths with the relativistic jets of different $\dot{E}_{\text {dep }}$.
\end{abstract}

Keywords. Galaxy: halo - gamma rays: bursts - nuclear reactions, nucleosynthesis, abundances - stars: abundances - stars: Population II - supernovae: general

\section{Introduction}

Among the most interesting recent developments in the study of supernovae (SNe) is the establishment of the Gamma-Ray Burst (GRB)-Supernova Connection (Woosley \& Bloom 2006). Three GRB-associated SNe have been observed so far: GRB 980425/ SN 1998bw (Galama et al. 1998, Iwamoto et al. 1998), GRB 030329/SN 2003dh (Stanek et al. 2003, Hjorth et al. 2003), and GRB 031203/SN 2003lw (Malesani et al. 2004). They are all very energetic supernovae, whose kinetic energy $E$ exceeds $10^{52} \mathrm{erg}$, more than 10 times the kinetic energy of normal core-collapse SNe. In the present paper, we use the term 'Hypernova (HN)' to describe such a hyper-energetic supernova with $E_{51}=E / 10^{51}$ erg $\gtrsim 10$ (Fig.1; Nomoto et al. 2004, Nomoto et al. 2006). The above three SNe associated with GRBs are called "GRB-HNe".

In contrast, "non-SN GRBs" (or dark HNe) have also been discovered (GRBs 060605 and 060614) (Fynbo et al. 2006, Gal-Yam et al. 2006, Della Valle et al. 2006, Gehrels et al. 2006). Upper limits to brightness of the possible SNe are about 100 times fainter than SN 1998bw. These correspond to upper limits to the ejected ${ }^{56} \mathrm{Ni}$ mass of $M\left({ }^{56} \mathrm{Ni}\right) \sim$ $10^{-3} M_{\odot}$ (see, e.g., Nomoto et al. 2007 for prediction). 


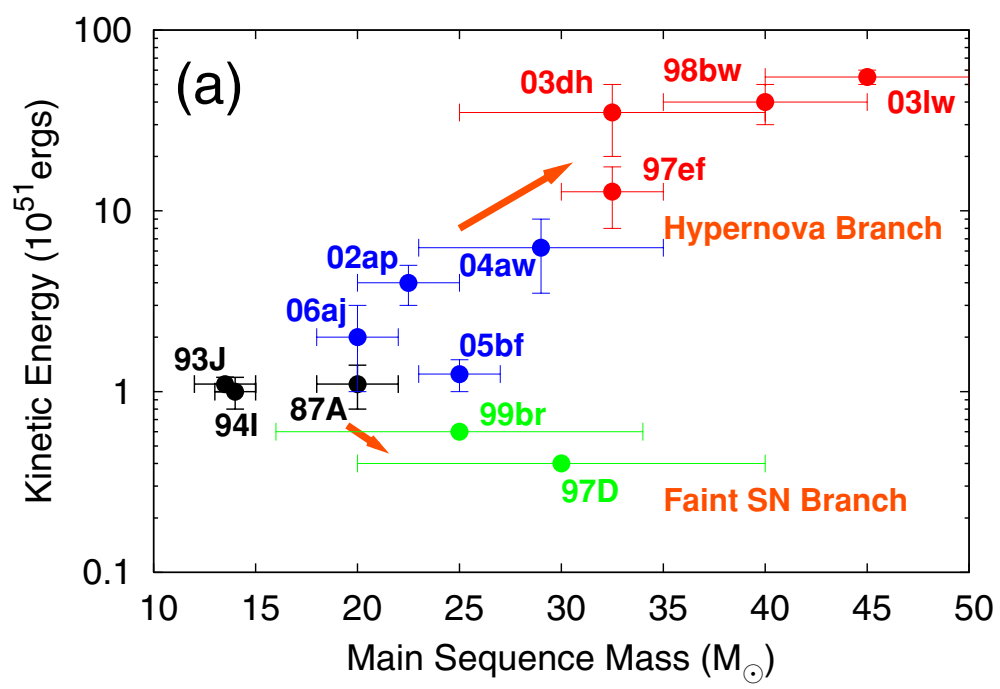

Figure 1. The kinetic explosion energy $E$ as a function of the main sequence mass $M$ of the progenitors for several supernovae/hypernovae. Hypernovae are the SNe with $E_{51}>10$.

Such hypernovae and GRBs are also likely to be hyper-aspherical explosions induced by relativistic jet(s) as suggested from photometric and spectroscopic observations.

We calculate nucleosynthesis in such hyper-energetic and hyper-aspherical explosions and find that resultant abundance features show some important differences from normal supernova explosions (e.g., Maeda et al. 2002, Maeda \& Nomoto 2003, Tominaga et al. 2007, Tominaga 2008). We show that such features can explain the peculiar abundance patterns observed in the extremely metal-poor (EMP), and hyper-metal-poor (HMP) halo stars (e.g., Hill, François, \& Primas 2005, Beers \& Christlieb 2005). This approach would lead to identifying the First Stars in the Universe, which is one of the important challenges of the current astronomy.

\section{Nucleosynthesis in Jet-Induced Explosions}

We calculate hydrodynamics and nucleosynthesis of the explosions induced by relativistic jets (jet-induced explosions) (Fig. 2) (Tominaga et al. 2007, Tominaga 2008). For the $40 M_{\odot}$ stars (Umeda \& Nomoto 2005, Tominaga, Umeda, \& Nomoto 2007), the jets are injected at a radius $R_{0} \sim 900 \mathrm{~km}$ (corresponding to an enclosed mass of $\left.M_{0} \sim 1.4 M_{\odot}\right)$. The most important parameter in our models is the energy deposition rate $\dot{E}_{\mathrm{dep}}$. We investigate the dependence of nucleosynthesis outcome on $\dot{E}_{\mathrm{dep}}$ for a range of $\dot{E}_{\mathrm{dep}, 51} \equiv \dot{E}_{\mathrm{dep}} / 10^{51} \mathrm{erg} \mathrm{s}^{-1}=0.3-1500$. The diversity of $\dot{E}_{\mathrm{dep}}$ is consistent with the wide range of the observed isotropic equivalent $\gamma$-ray energies and timescales of GRBs (Amati et al. 2006 and references therein). Variations of activities of the central engines, possibly corresponding to different rotational velocities or magnetic fields, may well produce the variation of $\dot{E}_{\mathrm{dep}}$.

The upper panel of Figure 3 shows the dependence of the ejected $M\left({ }^{56} \mathrm{Ni}\right)$ on $\dot{E}_{\mathrm{dep}}$. Generally, higher $\dot{E}_{\text {dep }}$ leads to the synthesis of larger $M\left({ }^{56} \mathrm{Ni}\right)$ in explosive nucleosynthesis because of higher post-shock densities and temperatures (e.g., Maeda \& Nomoto 2003, Nagataki et al. 2006). If $\dot{E}_{\mathrm{dep}, 51} \gtrsim 60$, we obtain $M\left({ }^{56} \mathrm{Ni}\right) \gtrsim 0.1 M_{\odot}$, which is consistent with the brightness of GRB-HNe. Some $\mathrm{C}+\mathrm{O}$ core materials are ejected along 


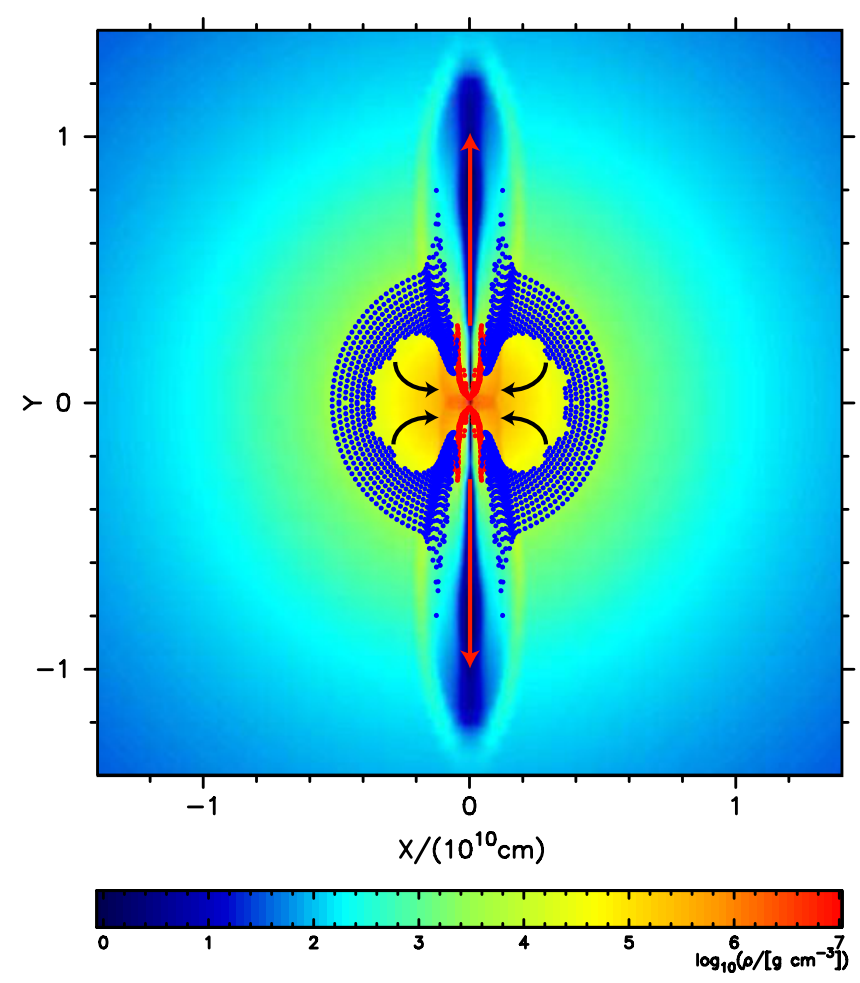

Figure 2. The density structure of the $40 M_{\odot}$ Pop III star explosion model of $\dot{E}_{\mathrm{dep}, 51}=15$ at $1 \mathrm{sec}$ after the start of the jet injection. The jets penetrate the stellar mantle (grey arrows) and material falls on the plane perpendicular to the jets (black arrows). The dots represent ejected Lagrangian elements dominated by $\mathrm{Fe}\left({ }^{56} \mathrm{Ni}\right.$, grey) and by $\mathrm{O}$ (black).

the jet-direction, but a large amount of materials along the equatorial plane fall back (Fig. 2).

For $\dot{E}_{\mathrm{dep}, 51} \gtrsim 60$, the remnant mass is initially $M_{\mathrm{rem}}^{\text {start }} \sim 1.5 M_{\odot}$ and grows as materials are accreted from the equatorial plane (Fig. 2). The final $\mathrm{BH}$ mass is generally larger for smaller $\dot{E}_{\mathrm{dep}}$. The final $\mathrm{BH}$ masses range from $M_{\mathrm{BH}}=10.8 M_{\odot}$ for $\dot{E}_{\mathrm{dep}, 51}=60$ to $M_{\mathrm{BH}}=5.5 M_{\odot}$ for $\dot{E}_{\mathrm{dep}, 51}=1500$, which are consistent with the observed masses of stellar-mass BHs (Bailyn et al. 1998). The model with $\dot{E}_{\mathrm{dep}, 51}=300$ synthesizes $M\left({ }^{56} \mathrm{Ni}\right) \sim 0.4 M_{\odot}$ and the final mass of $\mathrm{BH}$ left after the explosion is $M_{\mathrm{BH}}=6.4 M_{\odot}$.

For low energy deposition rates $\left(\dot{E}_{\mathrm{dep}, 51}<3\right)$, in contrast, the ejected ${ }^{56} \mathrm{Ni}$ masses $\left(M\left({ }^{56} \mathrm{Ni}\right)<10^{-3} M_{\odot}\right)$ are smaller than the upper limits for GRBs 060505 and 060614.

If the explosion is viewed from the jet direction, we would observe GRB without SN re-brightening. This may be the situation for GRBs 060505 and 060614 . In particular, for $\dot{E}_{\text {dep }, 51}<1.5,{ }^{56} \mathrm{Ni}$ cannot be synthesized explosively and the jet component of the Fepeak elements dominates the total yields (Fig. 4). The models eject very little $M\left({ }^{56} \mathrm{Ni}\right)$ $\left(\sim 10^{-6} M_{\odot}\right)$.

For intermediate energy deposition rates $\left(3 \lesssim \dot{E}_{\mathrm{dep}, 51}<60\right)$, the explosions eject $10^{-3} M_{\odot} \lesssim M\left({ }^{56} \mathrm{Ni}\right)<0.1 M_{\odot}$ and the final $\mathrm{BH}$ masses are $10.8 M_{\odot} \lesssim M_{\mathrm{BH}}<15.1 M_{\odot}$. The resulting $\mathrm{SN}$ is faint $\left(M\left({ }^{56} \mathrm{Ni}\right)<0.01 M_{\odot}\right)$ or sub-luminous $\left(0.01 M_{\odot} \lesssim M\left({ }^{56} \mathrm{Ni}\right)<\right.$ $\left.0.1 M_{\odot}\right)$. 


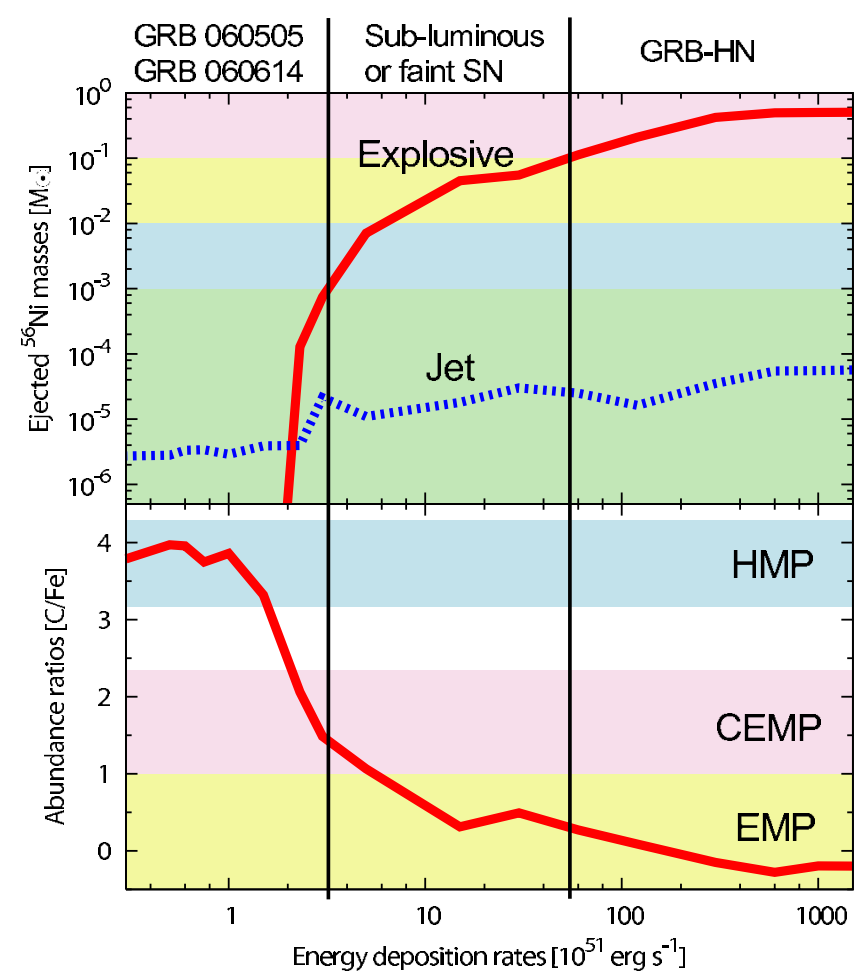

Figure 3. Upper: the ejected ${ }^{56} \mathrm{Ni}$ mass ( solid: explosive nucleosynthesis products, dashed: the jet contribution) as a function of the energy deposition rate. The background shows the corresponding SNe (top: GRB-HNe, top-middle: sub-luminous SNe, middle: faint SNe, bottom: GRBs 060505 and 060614). Vertical lines divide the resulting SNe according to their brightness. Lower: the dependence of abundance ratio $[\mathrm{C} / \mathrm{Fe}]$ on the energy deposition rate. The background color shows the corresponding metal-poor stars (bottom: EMP, middle: CEMP, top: HMP stars).

Table 1. Metal-poor stars.

\begin{tabular}{cccc}
\hline Name & {$[\mathrm{Fe} / \mathrm{H}]$} & Features & Reference \\
\hline HE 0107-5240 & -5.3 & C-rich, Co-rich?, $[\mathrm{Mg} / \mathrm{Fe}] \sim 0$ & Christlieb et al. 2002 \\
HE 1327-2326 & -5.5 & C, O, Mg-rich & Frebel et al. 2005 , Aoki et al. 2006 \\
HE 0557-4840 & -4.8 & C, Ca, Sc, Ti-rich, $[\mathrm{Co} / \mathrm{Fe}] \sim 0$ & Norris et al. 2007 \\
HE 1300+0157 & -3.9 & C, Si, Ca,Sc,Ti, Co-rich & Frebel et al. 2007 \\
HE 1424-0241 & -4.0 & Co,Mn-rich, Si,Ca,Cu-poor & Cohen et al. 2007 \\
CS 22949-37 & -4.0 & C,N,O,Mg,Co,Zn-rich & Depagne et al. 2002 \\
CS 29498-43 & -3.5 & C,N,O,Mg-rich, [Co/Fe] 0 & Aoki et al. 2004 \\
BS 16934-002 & -2.8 & O,Mg-rich, C-poor & Aoki et al. 2007 \\
\hline
\end{tabular}

\section{Abundance Patterns of Extremely Metal-Poor Stars}

Table 1 summarizes the abundance features of various EMP stars. In addition to HMP stars, we focus on the recently discovered first Ultra Metal-Poor (UMP) star (Norris et al. 2007) and the very peculiar Si-poor star (Cohen et al. 2007). Many of these EMP stars have high $[\mathrm{Co} / \mathrm{Fe}]$, suggesting the $\mathrm{HN}$-connection.

\subsection{C-rich Metal-Poor Stars (CEMP)}

The lower panel of Figure 3 shows the dependence of the abundance ratio $[\mathrm{C} / \mathrm{Fe}]$ on $\dot{E}_{\mathrm{dep}}$. Lower $\dot{E}_{\mathrm{dep}}$ yields larger $M_{\mathrm{BH}}$ and thus larger $[\mathrm{C} / \mathrm{Fe}]$, because the infall decreases the 


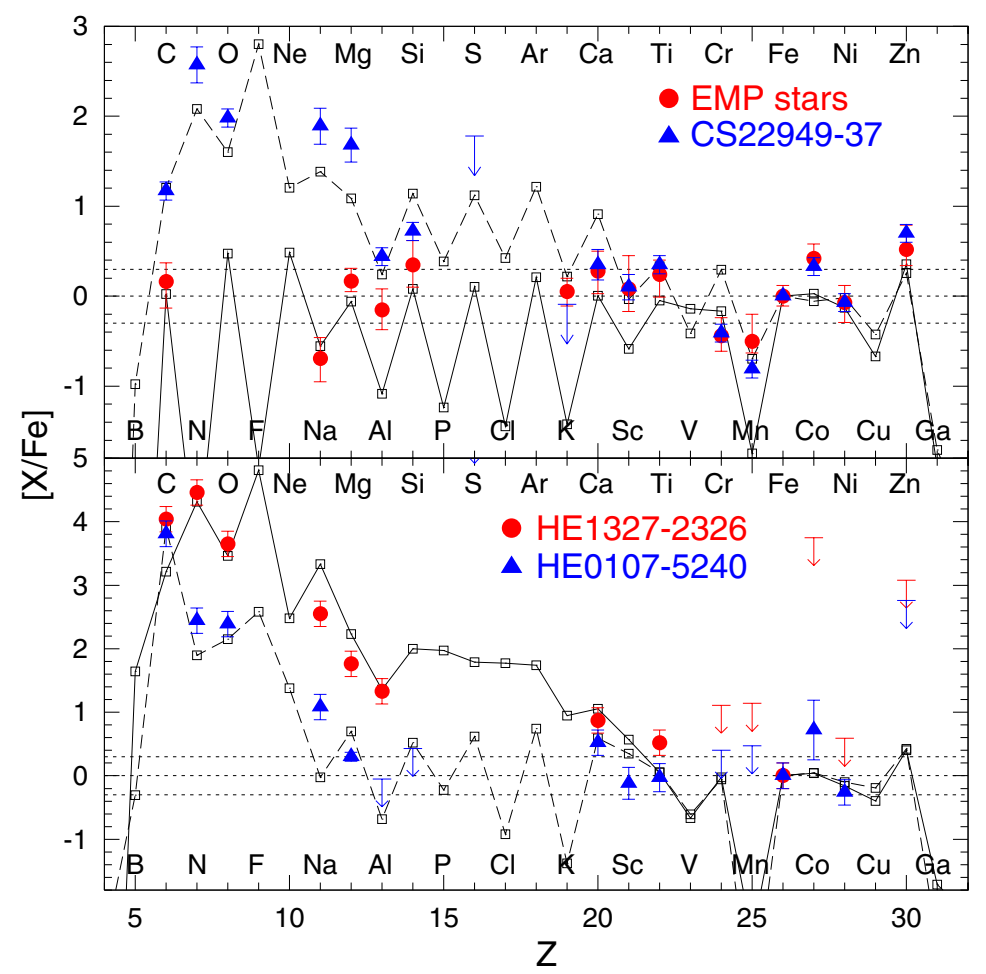

Figure 4. A comparison of the abundance patterns between the metal-poor stars and our models. Upper: typical EMP (dots, Cayrel et al. 2004) and CEMP (triangles, CS 22949-37, Depagne et al. 2002) stars and models with $\dot{E}_{\mathrm{dep}, 51}=120$ (solid line) and $=3.0$ (dashed line). Lower: HMP stars: HE 1327-2326, (dots, e.g., Frebel et al. 2005), and HE 0107-5240, (triangles, Christlieb et al. 2002, Bessell \& Christlieb 2005) and models with $\dot{E}_{\mathrm{dep}, 51}=1.5$ (solid line) and $=0.5$ (dashed line).

amount of inner core material (Fe) relative to that of outer material (C) (see also Maeda \& Nomoto 2003$)$. As in the case of $M\left({ }^{56} \mathrm{Ni}\right),[\mathrm{C} / \mathrm{Fe}]$ changes dramatically at $\dot{E}_{\mathrm{dep}, 51} \sim 3$.

The abundance patterns of the EMP stars are good indicators of SN nucleosynthesis because the Galaxy was effectively unmixed at $[\mathrm{Fe} / \mathrm{H}]<-3$ (e.g., Tumlinson 2006). They are classified into three groups according to $[\mathrm{C} / \mathrm{Fe}]$ :

(1) $[\mathrm{C} / \mathrm{Fe}] \sim 0$, normal EMP stars $(-4<[\mathrm{Fe} / \mathrm{H}]<-3$, e.g., Cayrel et al. 2004);

(2) $[\mathrm{C} / \mathrm{Fe}] \gtrsim+1$, Carbon-enhanced EMP $(\mathrm{CEMP})$ stars $(-4<[\mathrm{Fe} / \mathrm{H}]<-3$, e.g., CS 22949-37, Depagne et al. 2002);

(3) $[\mathrm{C} / \mathrm{Fe}] \sim+4$, hyper metal-poor $(\mathrm{HMP})$ stars $([\mathrm{Fe} / \mathrm{H}]<-5$, e.g., HE 0107-5240, Christlieb et al. 2002, Bessell \& Christlieb 2005; HE 1327-2326, Frebel et al. 2005).

Figure 4 shows that the abundance patterns of the averaged normal EMP stars, the CEMP star CS 22949-37, and the two HMP stars (HE 0107-5240 and HE 1327-2326) are well-reproduced by models with $\dot{E}_{\mathrm{dep}, 51}=120,3.0,1.5$, and 0.5 , respectively. The model for the normal EMP stars ejects $M\left({ }^{56} \mathrm{Ni}\right) \sim 0.2 M_{\odot}$, i.e., a factor of 2 less than SN 1998bw. On the other hand, the models for the CEMP and the HMP stars eject $M\left({ }^{56} \mathrm{Ni}\right) \sim 8 \times 10^{-4} M_{\odot}$ and $4 \times 10^{-6} M_{\odot}$, respectively, which are always smaller than the upper limits for GRBs 060505 and 060614 . The N/C ratio in the models for CS 2294937 and HE 1327-2326 is enhanced by partial mixing between the He and $\mathrm{H}$ layers during pre-supernova evolution (Iwamoto et al. 2005). 

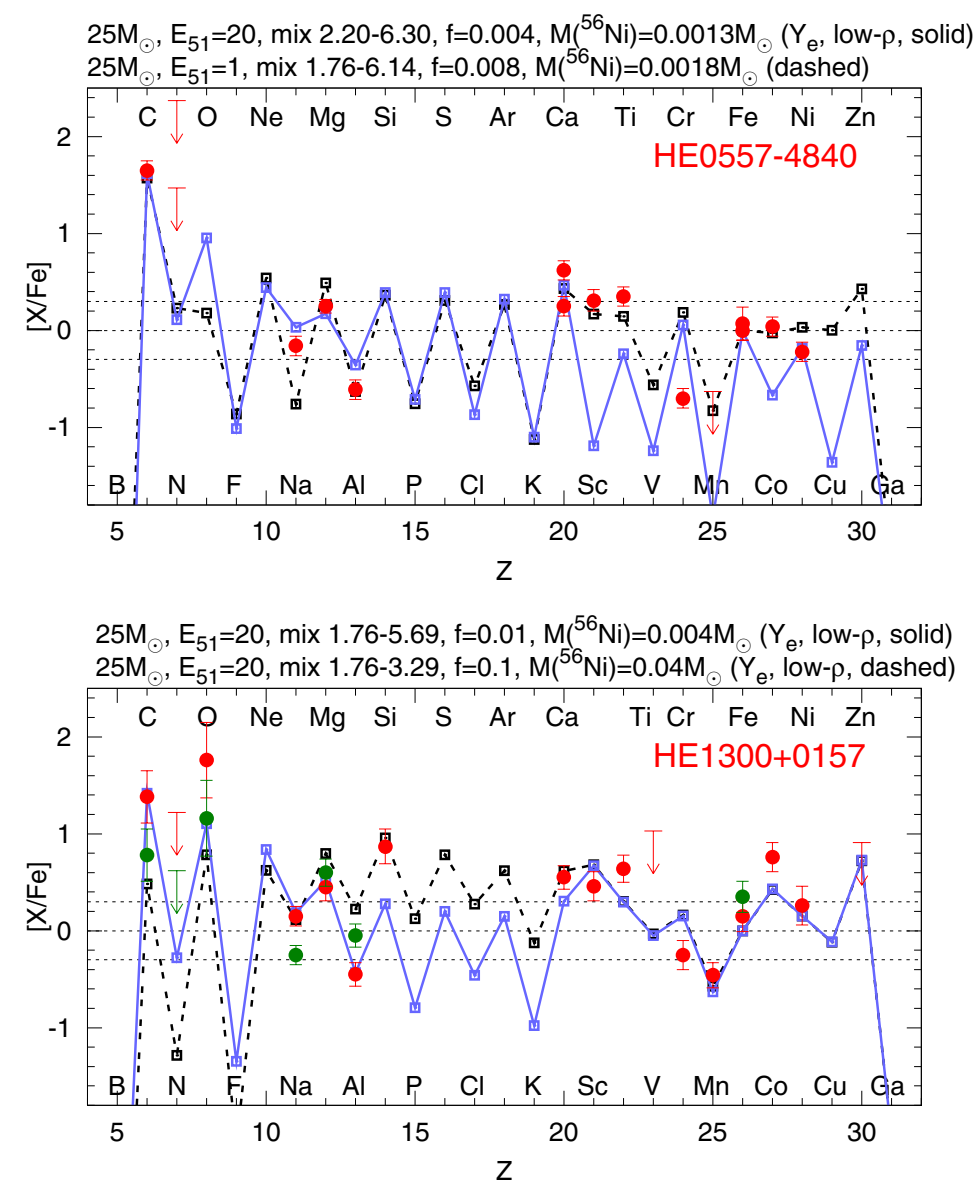

Figure 5. Comparisons of the abundance patterns between the mixing-fallback models and the UMP star HE0557-4840 (upper: Norris et al. 2007), and the CEP star HE1300+0157 (lower: Frebel et al. 2007).

\subsection{UMP Star HE 0557-4840 and CEMP-no Star HE 1300+0157}

The abundance pattern of the first Ultra Metal-Poor (UMP) star (HE 0557-4840: Norris et al. 2007$)$ is shown in Figure 5 and compared with the $\mathrm{HN}\left(E_{51}=20\right)$ and $\mathrm{SN}\left(E_{51}=1\right)$ models of the $25 M_{\odot}$ stars. The $\mathrm{Co} / \mathrm{Fe}$ ratio $([\mathrm{Co} / \mathrm{Fe}] \sim 0)$ requires a high energy explosion and the high $[\mathrm{Sc} / \mathrm{Ti}]$ and $[\mathrm{Ti} / \mathrm{Fe}]$ ratios require a high-entropy explosion. As shown in Figure 5 (upper), a HN model with a "low-density" modification (Tominaga et al. 2007) is in a good agreement with the abundance pattern of HE 0557-4840. The model indicates $M\left({ }^{56} \mathrm{Ni}\right) \sim 10^{-3} M_{\odot}$ being similar to faint SN models for CEMP stars. The [Cr/Fe] ratio in the model is much higher than that of HE 0557-4840.

The abundance pattern of the CEMP-no star (i.e., CEMP with no neutron capture elements) HE 1300+0157 (Frebel et al. 2007) is shown in Figure 5 (lower) and marginally reproduced by the hypernova model with $M_{\mathrm{MS}}=25 M_{\odot}$ and $E_{51}=20$. The large $[\mathrm{Co} / \mathrm{Fe}]$ particularly requires the high explosion energy.

\subsection{Si-Poor Star: HE 1424-0241}

The very peculiar Si-poor abundance pattern of HE 1424-0241 (Cohen et al. 2007) is shown in Figure 6 (upper) and compared with the model of $M_{\mathrm{MS}}=50 M_{\odot}$ and $E_{51}=40$. 

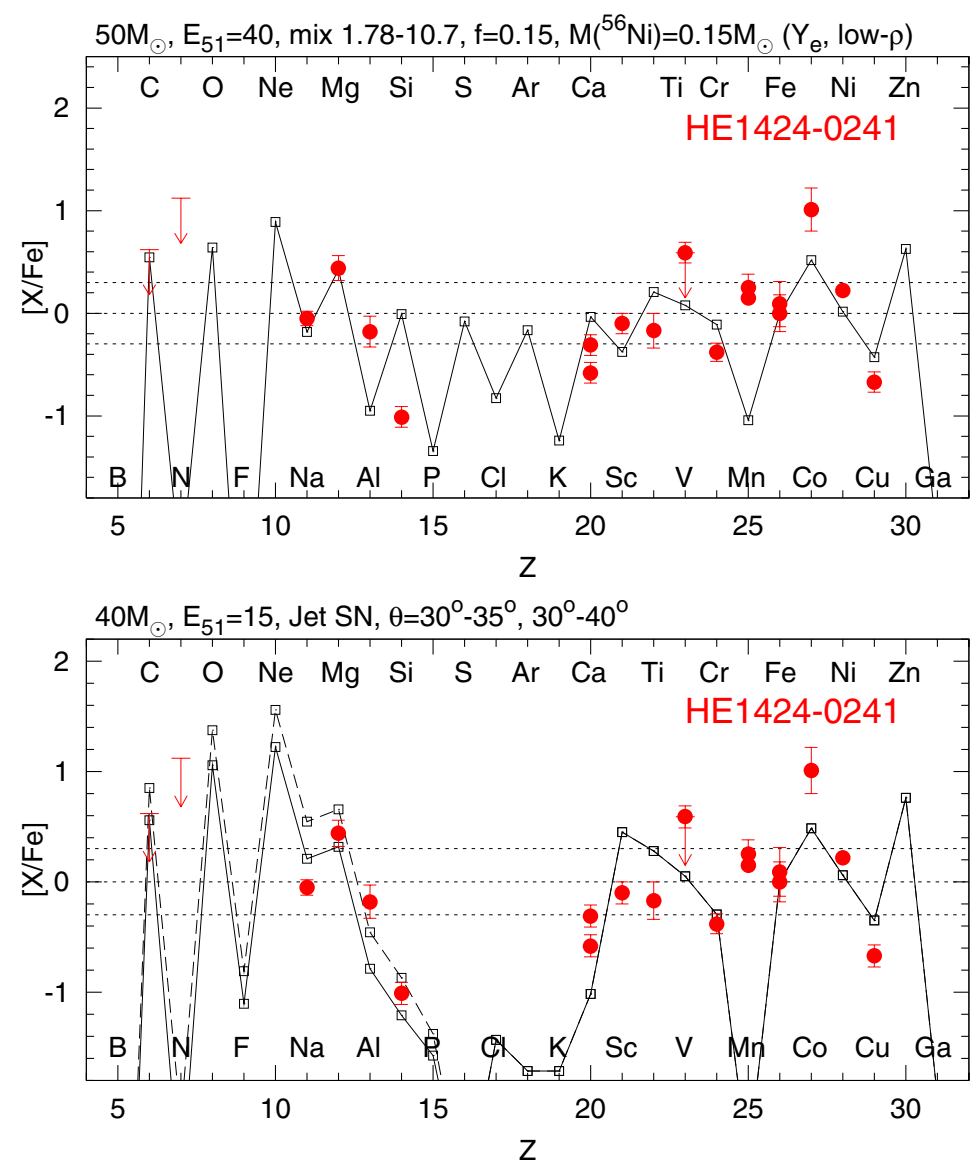

Figure 6. Comparisons between the abundance patterns of HE1424-0241 (Cohen et al. 2007) and the mixing-fallback model (upper), and the angle-delimited yields integrated over $30^{\circ}-40^{\circ}$ (dashed line) and $30^{\circ}-35^{\circ}$ (solid line) of the jet-induced SN model with $\dot{E}_{\mathrm{dep}}=1.2 \times 10^{53} \mathrm{erg} \mathrm{s}^{-1}$ (lower).

The high $[\mathrm{Mg} / \mathrm{Si}]$ ratio cannot be reproduced by this model. The peculiar abundance pattern of HE 1424-0241 is a challenge to the explosion models.

The angle-delimited yield provides a possibility to explain the high $[\mathrm{Mg} / \mathrm{Si}]$ and normal $[\mathrm{Mg} / \mathrm{Fe}]$. Figure 6 (lower) shows that the yields integrated over $30^{\circ}-40^{\circ}$ or $30^{\circ}-35^{\circ}$ reproduce the abundance pattern of $\mathrm{HE} 1424-0241$. The yields consist of $\mathrm{Mg}$ in the inner region and $\mathrm{Fe}$ in the outer region.

Thus the most difficult pattern can be reproduced by the angular dependence of the yield. The high $[\mathrm{Mg} / \mathrm{Si}]$ and normal $[\mathrm{Mg} / \mathrm{Fe}]$ are realized if the heavy elements penetrate into the stellar mantle and expand laterally (i.e., the duration of the jet injection is long) and if $\mathrm{Mg}$ along the equatorial plane is not accreted onto the central region (i.e., $\dot{E}_{\mathrm{dep}}$ is large).

\section{Concluding Remarks}

We show that (1) the explosions with large energy deposition rate, $\dot{E}_{\mathrm{dep}}$, are observed as GRB-HNe and their yields can explain the abundances of normal EMP stars, and (2) the explosions with small $\dot{E}_{\text {dep }}$ are observed as GRBs without bright SNe and can be 
responsible for the formation of the CEMP and the HMP stars. We thus propose that GRB-HNe and GRBs without bright SNe belong to a continuous series of BH-forming massive stellar deaths with the relativistic jets of different $\dot{E}_{\text {dep }}$. The very peculiar Si-poor EMP star can also be explained by the angle-delimited yield.

\section{References}

Amati, L., Della Valle, M., Frontera, F., et al. 2007, A\& $A, 463,913$

Aoki, W., Norris, J. E., Ryan, S. G., et al. 2004, ApJ, 608, 971

Aoki, W., Frebel A., Christlieb, N., et al. 2006, ApJ, 639, 897

Aoki, W., Honda, S., Beers, T. C., et al. 2007, ApJ, 660, 747

Bailyn, C. D., Jain, R. K., Coppi, P., \& Orosz, J. A. 1998, ApJ, 499, 367

Beers, T. \& Christlieb, N. 2005, ARA\&A, 43, 531

Bessell, M. S. \& Christlieb, N. 2005, in V. Hill, François, P., \& F. Primas (eds.), From Lithium to Uranium: Elemental Tracers of Early Cosmic Evolution, Proc. IAU Symposium No. 228 (Cambridge: Cambridge Univ. Press), 237

Cayrel, R., Depagne, E., Spite. M., et al. 2004, A\&A, 416, 1117

Christlieb, N., Bessell, M. S., Beers, T. C., et al. 2002, Nature, 419, 904

Cohen, J. G., McWilliam, A., Christlieb, N., et al. 2007, ApJ, 659, L161

Della Valle, M., Chincarini, G., Panagia, N., et al. 2006, Nature, 444, 1050

Depagne, E., Hill, V., Spite, M., et al. 2002, A\&A, 390, 187

Fynbo, J. P. U., Watson, D., Thöne, C. C., et al. 2006, Nature, 444, 1047

Frebel, A., Aoki, W., Christlieb, N., et al. 2005, Nature, 434, 871

Frebel, A., Norris, J. E., Aoki, W., et al. 2007, ApJ, 658, 534

Galama, T., Vreeswijk, P. M., van Paradijs, J., et al. 1998, Nature, 395, 670

Gal-Yam, A., Fox, D. B., Price, P. A., et al. 2006, Nature, 444, 1053

Gehrels, N., Norris, J. P., Barthelmy, S. D., et al. 2006, Nature, 444, 1044

Hill, V., François, P., \& Primas, F. (eds.) 2005, From Lithium to Uranium: Elemental Tracers of Early Cosmic Evolution, Proc. IAU Symp. No. 228 (Cambridge: Cambridge Univ. Press)

Hjorth, J., Sollerman, J., Møller, P., et al. 2003, Nature, 423, 847

Iwamoto, K., Mazzali, P. A., Nomoto, K., et al. 1998, Nature, 395, 672

Iwamoto, N., Umeda, H., Tominaga, N., et al. 2005, Science, 309, 451

Maeda, K., Nakamura, T., Nomoto, K., et al. 2002, ApJ, 565, 405

Maeda, K. \& Nomoto, K. 2003, ApJ, 598, 1163

Malesani, J., Tagliaferri, G., Chincarini, G., et al. 2004, ApJ, 609, L5

Nagataki, S., Mizuta, A., \& Sato, K. 2006, ApJ, 647, 1255

Nomoto, K., Maeda, K., Mazzali, P. A., et al., 2004, in: C. L. Fryer (ed.), Stellar Collapse (Dordrecht: Kluwer), Astrophys. Space Science Lib, 302, 277 (astro-ph/0308136)

Nomoto, K., Tominaga, N., Umeda, H., et al. 2006, Nuclear Phys A, 777, 424

Nomoto, K., Tominaga, N., Tanaka, M., et al. 2007, in: Swift and GRBs: Unveiling the Relativistic Universe, Nuovo Cimento B, 121, 1207 (astro-ph/0702472)

Norris, J. E., Crhistlieb, N., Korn, A. J., et al. 2007, ApJ, 670, 774

Stanek, K. Z., Matheson, T., Garnavich, P. M., et al. 2003, ApJ, 591, L17

Tominaga, N., Tanaka, M., Nomoto, K., et al. 2005, ApJ, 633, L97

Tominaga, N., Maeda, K., Umeda, H., et al. 2007, ApJ, 657, L77

Tominaga, N., Umeda, H., \& Nomoto, K. 2007, ApJ, 660, 516

Tominaga, N. 2008, ApJ, submitted (arXiv:0711.4815)

Tumlinson, J. 2006, ApJ, 641, 1

Umeda, H. \& Nomoto, K. 2002, ApJ, 565, 385

Umeda, H. \& Nomoto, K. 2005, ApJ, 619, 427

Woosley, S. E. \& Bloom, J. S. 2006, ARA\&A, 44, 507 